\title{
Standardisation of cardiopulmonary exercise testing in chronic lung diseases: summary of key findings from the ERS task force
}

\author{
Thomas Radtke (10) ${ }^{1}$, loannis Vogiatzis ${ }^{2,3,9}$, Don S. Urquhart (1) ${ }^{4}$, \\ Pierantonio Laveneziana ${ }^{5,6}$, Richard Casaburi ${ }^{7}$ and Helge Hebestreit ${ }^{8,9}$, on \\ behalf of the European Respiratory Society Task Force on Standardisation of \\ Cardiopulmonary Exercise Testing in Chronic Lung Diseases ${ }^{10}$
}

\begin{abstract}
Affiliations: ${ }^{1}$ Division of Occupational and Environmental Medicine, Epidemiology, Biostatistics and Prevention Institute, University of Zurich and University Hospital Zurich, Zurich, Switzerland. ${ }^{2}$ Dept of Sport, Exercise and Rehabilitation, Faculty of Health and Life Sciences, Northumbria University Newcastle, Newcastle, UK. ${ }^{3}$ First Dept of Respiratory Medicine, National and Kapodistrian University of Athens, Athens, Greece. ${ }^{4}$ Dept of Paediatric Respiratory and Sleep Medicine, Royal Hospital for Sick Children, Edinburgh, UK. ${ }^{5}$ Sorbonne Université, INSERM, UMRS1158 Neurophysiologie Respiratoire Expérimentale et Clinique, Paris, France. ${ }^{6}$ AP-HP Sorbonne Université, Groupe Hospitalier Pitié-Salpêtrière Charles Foix, Service des Explorations Fonctionnelles de la Respiration, de l'Exercice et de la Dyspnée du Département Médico-Universitaire "APPROCHES", Paris, France. ${ }^{7}$ Rehabilitation Clinical Trials Center, Los Angeles Biomedical Research Institute at Harbor-UCLA Medical Center, Torrance, CA, USA. ${ }^{8}$ Paediatric Dept, University Hospital Würzburg, Würzburg, Germany. ${ }^{9}$ Task force co-chairs. ${ }^{10} \mathrm{~A}$ full list of members of the task force can be found in the acknowledgements section.
\end{abstract}

Correspondence: Helge Hebestreit, Universitäts-Kinderklinik, Josef-Schneider-Str. 297080 Würzburg, Germany, E-mail: hebestreitduni-wuerzburg.de

@ERSpublications

The new ERS statement facilitates standardisation of conducting, reporting and interpreting cardiopulmonary exercise tests in chronic lung diseases. The conclusions are especially valuable for multicentre comparisons and for establishing global reference data. http://bit.ly/32Y0eO0

Cite this article as: Radtke T, Vogiatzis I, Urquhart DS, et al. Standardisation of cardiopulmonary exercise testing in chronic lung diseases: summary of key findings from the ERS task force. Eur Respir J 2019; 54: 1901441 [https://doi.org/10.1183/13993003.01441-2019].

\begin{abstract}
Introduction
Cardiopulmonary exercise testing (CPET) is a powerful diagnostic and prognostic tool in the management of people with a variety of chronic lung diseases [1-5]. Despite the remarkable increase in the number of publications on CPET over the past decades [2], CPET remains underutilised in the field of respiratory medicine. Numerous challenges of utilising CPET in both clinical practice and research await resolution, including the establishment of global reference values, in line with those available for lung function and pulmonary diffusing capacity [6, 7]. Prior to such an international effort, strict standardisation of testing procedures is indispensable, in order to obtain valid and reliable results and to enable interpretation of CPET results within and between centres and individuals. Furthermore, an internationally standardised protocol is a prerequisite for the use of CPET data in patient registries and in retrospective collaborative research.
\end{abstract}


Previous statements by the European Respiratory Society (ERS) [8-10] and the American Thoracic Society (ATS) and the American College of Chest Physicians (ACCP) [11] partly address standardisation, but none of these statements included information on the standardisation of CPET procedures with regard to a range of lung diseases, nor considered the patient's experience of CPET. As a consequence, various protocol modifications are used across the world, which may impact the choice of reference data and interpretation. The aim of this task force was to provide a strict technical standardisation of CPET procedures that could be applied to multiple respiratory conditions.

An ERS task force of adult and paediatric physicians, physiologists, physiotherapists, exercise scientists and methodologists with expertise in CPET across a wide range of lung diseases conducted a rigorous systematic literature review to provide a comprehensive account of published CPET procedures that could be uniformly applied across different settings, such as research and/or clinical practice. Importantly, to gather patients' perspectives on CPET, an online survey supported by the European Lung Foundation (ELF) was conducted.

The systematic literature review identified 7914 studies, of which 595 studies with 26523 patients were included in the analysis [12]. Among included studies, the most common lung diseases were COPD and emphysema (54\%), cystic fibrosis (13\%), pulmonary arterial hypertension (11\%) and lung cancer (7\%). Whenever the literature review could not inform the task force on specific questions, existing guidelines and technical standards were used [8-11].

\section{“Ramp or minute-by-minute protocols are best suited for CPET in chronic lung diseases"}

As already recommended in previous statements by the ERS and ATS/ACCP $[8,11]$, incremental exercise protocols with stages of up to 1 min maximum are best to assess people with chronic lung diseases, since such protocols not only allow the measurement of peak values, but are preferred to evaluate important variables during submaximal exercise stages such as the ventilatory anaerobic threshold, the slope of the minute ventilation-carbon dioxide output relationship and the slope of the oxygen uptake-work rate relationship. If stages are longer, the adjustments to the change in work rate will be visible in the ventilatory and gas exchange data, thereby reducing the precision of determining the above CPET outcomes. Overall, the percentage of studies employing 1-min work rate increments was higher compared to ramp protocols for cycle and treadmill exercise testing (figure 1). While there was a clear preference for minute-by-minute cycle ergometer testing protocols in studies with COPD and cystic fibrosis patients, a protocol preference was less clear for other lung diseases. Reasons for protocol choices could not be systematically evaluated from the majority of original studies.

\section{"Cycle ergometry is the preferred testing modality across various lung diseases"}

Given the above considerations, cycle ergometry (92\% of studies) compared to treadmill ergometry ( $8 \%$ of studies) was the preferred testing modality across a wide range of lung diseases (figure 1).

\section{"Standardised exercise protocol is available"}

To be clinically feasible and acceptable to patients and staff, a CPET protocol should be as short as possible. Based on physiological considerations, the rigorous evidence-focused literature review, the clinical experience of the task force members, and patient survey responses, the task force recommends a protocol

FIGURE 1 Number of publications reporting use of cycle ergometry versus treadmill exercise protocols: results from the systematic literature review.

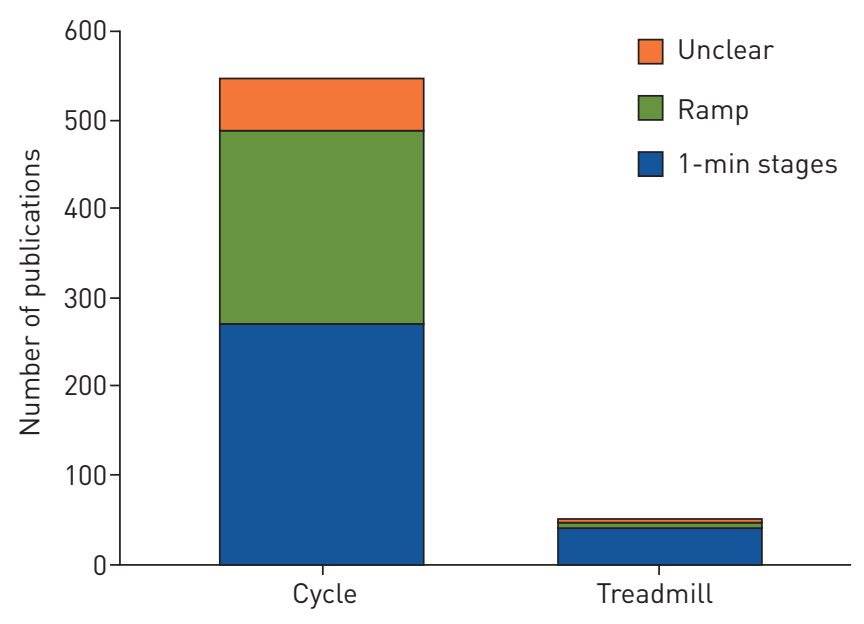


consisting of a resting phase lasting at least $3 \mathrm{~min}$, a 3-min "unloaded" phase at the lowest work rate provided by the cycle ergometer or treadmill, an 8- to 12-min incremental phase with equal work rate increments every minute, followed by a recovery phase of at least 2-3 min. Thus, the previously suggested testing protocol [8-11] was further concretised.

The task force also aimed to establish disease-specific work rate increments using an approach similar to that of WASSERMAn et al. [13]. However, work rate increments have proved difficult to estimate due to the lack of reported patient datasets and heterogeneity of lung diseases that clearly limits the use of the work rate increment estimations for clinical practice. Previous studies have provided work rate increment estimation for cycle ergometry for people with COPD [14], cystic fibrosis [15], asbestosis, silicosis and asthma [16], and for treadmill testing for healthy adults [17, 18] and COPD patients [19, 20]. Other, more general recommendations for the selection of work rate increments are based on estimated peak oxygen uptake $\left(V_{\mathrm{O}_{2}}\right.$ peak) and $V_{\mathrm{O}_{2}}$ during unloaded pedalling [8]. Based on the data available from the literature review, additional guidance is given for the selection of minute-by-minute increments in work rate in the full technical standard document [12].

\section{“Improved reporting of CPET methodology in future publications is prioritised"}

The rigorous systematic literature review identified a large heterogeneity and substantial lack of information with respect to the quality of reporting on CPET equipment, testing protocols and criteria to determine maximal effort in the original publications [12]. Based on a lack of sufficient information, the task force made suggestions regarding the operational dataset to be reported in future studies in order to achieve standardisation of CPET in lung diseases.

Of studies reporting CPET protocol details for cycle ergometry, only about 42\%, 58\% and 10\% provided information on the duration of the resting phase, unloaded phase and recovery phase, respectively. For treadmill ergometry, protocol information was given even less often (14\%,39\% and $8 \%)$. The selection of work rate increments for cycle and treadmill exercise testing was rarely reported in the original studies. About $8-10 \%$ of all included studies did not provide any information on the chosen work rate increment.

\section{“Interpretation of CPET is based on comprehensive review of available information"}

In general, the interpretation of CPET results requires the integration of testing results with the patient's medical history, and other clinical findings and investigations. The results of an exercise test may prompt further clinical investigations (e.g. vascular assessment, assessment of respiratory or peripheral muscle function). In this context, feedback from the patient, including reason for exercise termination, can be useful in evaluating exercise limitation. The task force report [12] provides a description and algorithm of how task force members interpret CPET results.

\section{“Patient survey participants perceive exercise testing as beneficial and suggest annual testing as optimal"}

With support from the ELF, the task force conducted an online survey available in nine languages to assess patients' perspectives, in order to gain insights into the perceived relevance and burden of taking part in CPET-related investigations. 295 patients from 34 countries responded to the survey. The majority were patients with COPD (26\%), sarcoidosis $(18 \%)$, cystic fibrosis $(16 \%)$ or asthma (13\%). Responders were asked about the benefits of taking part in CPET, with 75\% reporting improved knowledge about lung function as the greatest benefit. Many patients perceived the CPET as a highly beneficial part of their

FIGURE 2 Reported frequency of participating in cardiopulmonary exercise testing in the future: results from the patient survey.

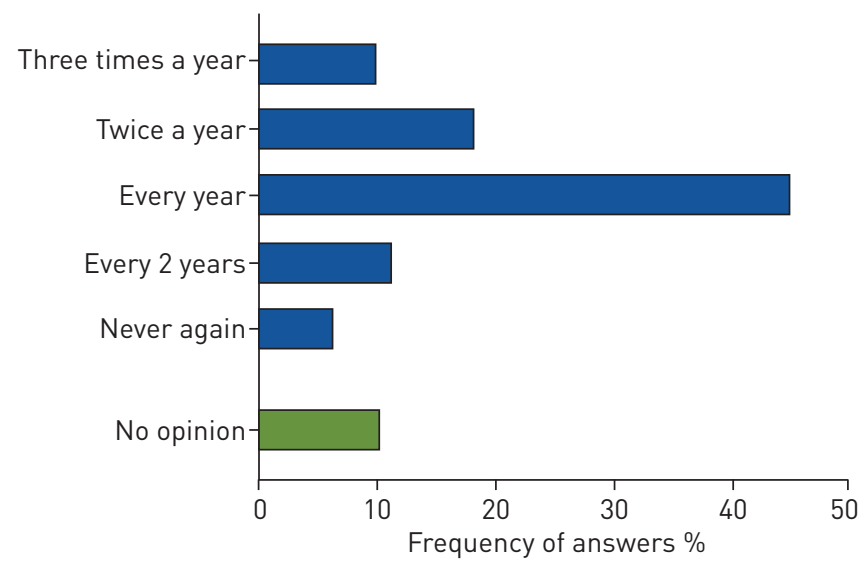


check-up and provided suggestions that informed this ERS task force [12]. For example, responders wished to perform CPET in a quieter environment, and have the opportunity to sip water or to use a fan during the recovery period. In addition, patients highlighted the need for more information about the testing procedures and improved individual care during the test. Such information is highly relevant and might be incorporated by people conducting and supervising CPET in their daily work. When asked how much of a problem various aspects of CPET were, responders rated as "serious problems": dryness in mouth (11\%), muscle soreness (10\%), bicycle seat discomfort (9\%), coughing (9\%) and mouthpiece discomfort (8\%).

The preferred frequency for participating in CPET reported by nearly $75 \%$ of respondents was at least annually (figure 2).

\section{Future research directions}

The task force identified important gaps with respect to CPET protocol standardisation [12]. Future research should focus on the standardisation of work rate increments for different lung diseases and the collection of large CPET datasets to establish normal values for relevant CPET outcomes in healthy subjects across age, including both female and male subjects in sufficient numbers and a wide range of ethnicities. Ideally this should occur in a collaborative effort between the ERS and the Global Lung Function Initiative [6, 7]. Moreover, future work should address the measurement properties of CPET using appropriate analytical methods in patients with various lung conditions and across a broad range of disease severity. Finally, this task force identified a clear lack of studies comparing different CPET procedures (e.g. cycling versus treadmill; impact of inspiratory capacity manoeuvres) on exercise testing results.

Acknowledgements: The authors are very grateful for the support of all members of this task force. We kindly thank the European Lung Foundation for their support with the Patient Survey and all survey respondents for their time and participation.

The task force on standardisation of cardiopulmonary exercise testing in chronic lung diseases consisted of: Thomas Radtke, Division of Occupational and Environmental Medicine, Epidemiology, Biostatistics and Prevention Institute, University of Zurich and University Hospital Zurich, Zurich Switzerland; Sarah Crook, Epidemiology, Biostatistics and Prevention Institute, University of Zurich, Zurich, Switzerland; Georgios Kaltsakas, Lane Fox Respiratory Service, St Thomas' Hospital, Guy's and St Thomas' NHS Foundation Trust, London, UK and First Dept of Respiratory Medicine, National \& Kapodistrian University of Athens, Greece; Zafeiris Louvaris, Faculty of Kinesiology and Rehabilitation Sciences, Division of Respiratory Rehabilitation, Dept of Rehabilitation Sciences KU Leuven, University Hospital Leuven, Leuven, Belgium; Danilo C. Berton, Unidade de Fisiologia Pulmonar, Hospital de Clínicas de Porto Alegre, Universidade Federal do Rio Grande do Sul, Brazil; Don S. Urquhart, Dept of Paediatric Respiratory and Sleep Medicine, Royal Hospital for Sick Children, Edinburgh, UK; Asterios Kampouras, Paediatric Dept, 424 General Military Hospital, Thessaloniki, Greece; Roberto A. Rabinovich, ELEGI Colt Laboratory, MRC Centre for Inflammation Research, The Queen's Medical Research Institute, University of Edinburgh, UK and Respiratory Medicine Dept, Royal Infirmary of Edinburgh, UK; Samuel Verges, HP2 Laboratory, Grenoble Alpes University, INSERM, Grenoble, France; Dimitris Kontopidis, Hellenic Cystic Fibrosis Association, Athens, Greece; Jeanette Boyd, European Lung Foundation (ELF), Sheffield, UK; Thomy Tonia, Institute of Social and Preventive Medicine, University of Bern, Bern, Switzerland; Daniel Langer, Faculty of Kinesiology and Rehabilitation Sciences, Dept of Rehabilitation Sciences, Research Group for Cardiovascular and Respiratory Rehabilitation, KU Leuven - University of Leuven, Belgium and Respiratory Rehabilitation and Respiratory Division, University Hospital Leuven, Leuven, Belgium; Jana De Brandt, REVAL Rehabilitation Research Center, BIOMED - Biomedical Research Institute, Faculty of Rehabilitation Sciences, Hasselt University, Diepenbeek, Belgium; Yvonne M.J. Goërtz, Dept of Research and Education, CIRO+, Centre of Expertise for Chronic Organ Failure, Horn, The Netherlands; Chris Burtin, REVAL - Rehabilitation Research Center, BIOMED Biomedical Research Institute, Faculty of Rehabilitation Sciences, Hasselt University, Diepenbeek, Belgium; Martijn A. Spruit, REVAL - Rehabilitation Research Center, BIOMED - Biomedical Research Institute, Faculty of Rehabilitation Sciences, Hasselt University, Diepenbeek, Belgium and Dept of Research and Education, CIRO+, Centre of Expertise for Chronic Organ Failure, Horn, The Netherlands and Dept of Respiratory Medicine, Maastricht University Medical Centre, NUTRIM School of Nutrition and Translational Research in Metabolism, Maastricht, The Netherlands; Dionne C.W. Braeken, Dept of Research and Education, CIRO+, Centre of Expertise for Chronic Organ Failure, Horn, The Netherlands; Sauwaluk Dacha, Faculty of Movement and Rehabilitation Sciences, Dept of Rehabilitation Sciences, Research Group for Cardiovascular and Respiratory Rehabilitation, KU Leuven - University of Leuven, Belgium and Respiratory Rehabilitation and Respiratory Division, University Hospital Leuven, Leuven, Belgium and Dept of Physical Therapy, Faculty of Associated Medical Sciences, Chiang Mai University, Chiang Mai, Thailand; Frits M.E. Franssen, Dept of Research and Education, CIRO+, Centre of Expertise for Chronic Organ Failure, Horn, The Netherlands and Dept of Respiratory Medicine, Maastricht University Medical Centre, NUTRIM School of Nutrition and Translational Research in Metabolism, Maastricht, The Netherlands; Pierantonio Laveneziana, Sorbonne Université, INSERM, UMRS1158 Neurophysiologie Respiratoire Expérimentale et Clinique, Paris, France and AP-HP Sorbonne Université, Groupe Hospitalier Pitié-Salpêtrière Charles Foix, Service des Explorations Fonctionnelles de la Respiration, de l'Exercice et de la Dyspnée du Département Médico-Universitaire "APPROCHES", Paris, France; Ernst Eber, Division of Paediatric Pulmonology and Allergology, Dept of Paediatrics and Adolescent Medicine, Medical University of Graz, Austria; Thierry Troosters, Dept of Rehabilitation Sciences, KU Leuven, Leuven, Belgium and Pulmonary Rehabilitation, University Hospital Gasthuisberg, Leuven, Belgium; J. Alberto Neder, Laboratory of Clinical Exercise Physiology \& Respiratory Investigation Unit, Kingston Health Science Center, Queen's University, Kingston, ON, Canada; Milo A. Puhan, Epidemiology, Biostatistics and Prevention Institute, University of Zurich, Zurich, Switzerland; Richard Casaburi, Rehabilitation Clinical Trials Center, Los Angeles Biomedical Research Institute at Harbor-UCLA Medical Center, Torrance, CA, USA; Ioannis Vogiatzis, Dept of Sport, Exercise and Rehabilitation, Faculty of Health 
and Life Sciences, Northumbria University Newcastle, UK and First Dept of Respiratory Medicine, National and Kapodistrian University of Athens, Greece; Helge Hebestreit, Paediatric Dept, University Hospital Würzburg, Würzburg, Germany.

Support statement: Funding was received from the European Respiratory Society, grant number TF-2016-12. Funding information for this article has been deposited with the Crossref Funder Registry.

Conflict of interest: T. Radtke reports personal fees for registration, travel/accommodation for conferences from Vifor Pharma, outside the submitted work. I. Vogiatzis has nothing to disclose. D.S. Urquhart has nothing to disclose. P. Laveneziana reports personal fees from Novartis France and Boehringer France, outside the submitted work. R. Casaburi reports that he shares patent US 7927251 B1, relating to a linear treadmill testing protocol, issued to Los Angeles Biomedical Research Institute. H. Hebestreit reports grants and personal fees for advisory board work from Vertex Pharmaceuticals, grants from Mukoviszidose e.V. (German CF Organisation), outside the submitted work; and is a coordinator of the European Cystic Fibrosis Society Exercise Working Group.

\section{References}

1 Hebestreit H, Hulzebos EHJ, Schneiderman JE, et al. Prognostic Value of CPET in CF Study Group. Cardiopulmonary exercise testing provides additional prognostic information in cystic fibrosis. Am J Respir Crit Care Med 2019; 199: 987-995.

2 Neder JA, Laveneziana P, Ward SA, et al. Introduction: CPET in clinical practice. Recent advances, current challenges and future directions. In: Palange P, Laveneziana P, Neder JA, et al., eds. Clinical Exercise Testing (ERS Monograph). Sheffield, European Respiratory Society, 2018.

3 O'Donnell D, Elbehairy A, Berton D, et al. Exercise testing in the evaluation of pharmacotherapy in COPD. In: Palange P, Laveneziana P, Neder JA, et al., eds. Clinical Exercise Testing (ERS Monograph). Sheffield, European Respiratory Society, 2018; pp. 235-250.

4 Laviolette L, Laveneziana P. Exercise testing in the prognostic evaluation of patients with lung and heart diseases. In: Palange P, Laveneziana P, Neder JA, et al., eds. Clinical Exercise Testing (ERS Monograph). Sheffield, European Respiratory Society, 2018; pp. 222-234.

5 Wensel R, Francis DP, Meyer FJ, et al. Incremental prognostic value of cardiopulmonary exercise testing and resting haemodynamics in pulmonary arterial hypertension. Int J Cardiol 2013; 167: 1193-1198.

6 Quanjer PH, Stanojevic S, Cole TJ, et al. Multi-ethnic reference values for spirometry for the 3-95-yr age range: the global lung function 2012 equations. Eur Respir J 2012; 40: 1324-1343.

7 Stanojevic S, Graham BL, Cooper BG, et al. Official ERS technical standards: Global Lung Function Initiative reference values for the carbon monoxide transfer factor for Caucasians. Eur Respir J 2017; 50: 1700010.

8 Roca J, Whipp BJ, Agusti AGN, et al. Clinical exercise testing with reference to lung diseases: indications, standardization and interpretation strategies. Eur Respir J 1997; 10: 2662-2689.

9 Palange P, Ward SA, Carlsen $\mathrm{KH}$, et al. Recommendations on the use of exercise testing in clinical practice. Eur Respir J 2007; 29: 185-209.

10 Puente-Maestu L, Palange P, Casaburi R, et al. Use of exercise testing in the evaluation of interventional efficacy: an official ERS statement. Eur Respir J 2016; 47: 429-460.

11 ATS/ACCP Statement on cardiopulmonary exercise testing. Am J Respir Crit Care Med 2003; 167: 211-277.

12 Radtke T, Crook S, Kaltsakas G, et al. ERS statement on standardisation of cardiopulmonary exercise testing in chronic lung diseases. Eur Respir Rev 2019; 28: 180101.

13 Wasserman K, Hansen JE, Sue DY, et al. Principles of Exercise Testing and Interpretation. 1st Edn. Philadelphia, Lea \& Febiger, 1986

14 Hartman JE, Slebos DJ, Boezen HM, et al. Selecting the increment size for a maximal incremental cycle test in patients with COPD. Respirology 2015; 20: 352-355.

15 Hulzebos HJ, Werkman MS, van Brussel M, et al. Towards an individualized protocol for workload increments in cardiopulmonary exercise testing in children and adolescents with cystic fibrosis. J Cyst Fibros 2012; 11: 550-554.

16 Ochmann U, Kotschy-Lang N, Raab W, et al. Is an individual prediction of maximal work rate by 6-minute walk distance and further measurements reliable in male patients with different lung diseases? Respiration 2013; 86: 384-392.

17 Myers J, Buchanan N, Walsh D, et al. Comparison of the ramp versus standard exercise protocols. J Am Coll Cardiol 1991; 17: 1334-1342.

18 Porszasz J, Casaburi R, Somfay A, et al. A treadmill ramp protocol using simultaneous changes in speed and grade. Med Sci Sport Exer 2003; 35: 1596-1603.

19 Holm SM, Rodgers W, Haennel RG, et al. Effect of modality on cardiopulmonary exercise testing in male and female COPD patients. Resp Physiol Neurobiol 2014; 192: 30-38.

20 Hsia D, Casaburi R, Pradhan A, et al. Physiological responses to linear treadmill and cycle ergometer exercise in COPD. Eur Respir J 2009; 34: 605-615. 